\title{
Polytropic spheres with electric charge: compact stars, the Oppenheimer-Volkoff and Buchdahl limits, and quasiblack holes
}

\author{
José D. V. Arbañil, ${ }^{1, *}$ José P. S. Lemos, ${ }^{2, \dagger}$ and Vilson T. Zanchin ${ }^{1, \ddagger}$ \\ ${ }^{1}$ Centro de Ciências Naturais e Humanas, Universidade Federal do ABC, \\ Rua Santa Adélia 166, 09210-170 Santo André, SP, Brazil. \\ ${ }^{2}$ Centro Multidisciplinar de Astrofísica - CENTRA, Departamento de Física, \\ Instituto Superior Técnico - IST, Universidade de Lisboa - UL, \\ Avenida Rovisco Pais 1, 1049-001 Lisboa, Portugal.
}

\begin{abstract}
We explore a class of compact charged spheres made of a charged perfect fluid with a polytropic equation of state. The charge density is chosen to be proportional to the energy density. The study is performed by solving the Tolman-Oppenheimer-Volkoff (TOV) equation which describes the hydrostatic equilibrium. We show the dependence of the structure of the spheres for several characteristic values of the polytropic exponent and for different values of the charge densities. We also study other physical properties of the charged spheres, such as the total mass, total charge, radius and sound speed and their dependence on the polytropic exponent. We find that for the polytropic exponent $\gamma=4 / 3$ the Chandrasekhar mass limit coincides with the Oppenheimer-Volkoff mass limit. We test the Oppenheimer-Volkoff limit for such compact objects. We also analyze the Buchdahl limit for these charged polytropic spheres, which happens in the limit of very high polytropic exponents, i.e., for a stiff equation of state. It is found that this limit is extremal and it is a quasiblack hole.
\end{abstract}

\section{INTRODUCTION}

\subsection{Compact stars and the TOV equation and method}

\subsubsection{Compact stars and the Oppenheimer-Volkoff limit}

Eddington, in discussing the internal constitution of stars, understood that by carefully choosing the temperature distribution along a given star, the gas could be made to obey a polytropic relation between its hydrostatic pressure $p$ and mass density $\rho$, namely, $p=\omega \rho^{\gamma}$, where $\omega$ and $\gamma$ are replaceable constants [1]. Following the tradition of calling spherically symmetric star models as gas spheres, he called these particular models polytropic gas spheres. The stars in question are stars like the sun, supported against gravitational collapse by matter and radiation pressure. By obtaining a consistent picture, his initial model for the stars was vindicated. On the other hand, when he and others tried to discuss the structure of white dwarfs, stars with much higher density and compact, they got it all wrong.

Chandrasekhar $[2,3]$ showed then definitely that white dwarfs are compact stars in which the pressure support against collapse comes from the quantum degeneracy of the electrons. The temperature throughout the star is negligible for its structure, and thus these stars, besides being compact, are also cold. In turn, this means there is an effective simple polytropic equation between pressure and density. Moreover, as the configuration gets more compact, the electrons get more and more relativistic and

\footnotetext{
*Electronic address: jose.arbanil@ufabc.edu.br

$\dagger$ Electronic address: joselemos@ist.utl.pt

${ }^{\ddagger}$ Electronic address: zanchin@ufabc.edu.br
}

the last equilibrium configuration is a star with a definite mass shrank to zero radius. This mass is called the Chandrasekhar limit, generally taken as $1.44 M_{\odot}[2,3]$.

Now, any given mass in zero radius should be treated in the context of general relativity. This was done by Tolman [4] and Oppenheimer and Volkoff [5] who showed that equilibrium configurations with neutrons, the neutron stars, much more compact than white dwarfs, could also be achieved, and that these neutron stars have again a mass limit, called the Oppenheimer-Volkoff limit. Whereas the Chandrasekhar limit appears within Newtonian gravitation when joined to relativistic kinematic effects in the degenerate matter, the Oppenheimer-Volkoff limit is a pure general relativistic limit. It appears because the pressure that supports the star also has an energy associated with it. When the pressure is sufficiently large, as for instance in a very compact neutron star, the pressure contribution to mass-energy makes the gravitational field large enough that it cannot be supported by the pressure itself. From then on, the star is unstable to collapse, giving the Oppenheimer-Volkoff limit. Through heuristic arguments, Landau had also found the Chandrasekhar and the Oppenheimer-Volkoff limit [6].

The question to what happen to stars that get more massive, or more compact, than allowed by the Oppenheimer-Volkoff limit was answered by Oppenheimer and Snyder [7] who showed that totally collapsed configurations, black holes, form. These are objects with a central singularity that somehow contains what was the star's matter, and with an event horizon from inside which nothing can escape.

The basic theory for white dwarfs, neutron stars and black holes was thus laid down in the decade of 1930. 


\subsubsection{The TOV equation and method and the structure of compact stars}

In their work, Tolman [4] and specially Oppenheimer and Volkoff [5] devised a method to find solutions in a consistent manner, most prone to numerical integration. They managed to put the structure equation for cold matter, i.e., matter with zero or negligible temperature, as $d p / d r=-(p+\rho)\left(4 \pi p r+m / r^{2}\right) /(1-2 m / r)$, where $p=p(r)$ is the pressure as a function of the radius $r, \rho(r)$ is the energy density, $m(r)$ is the mass function given by the equation $d m / d r=4 \pi r^{2} \rho$. This equation for the pressure is the Tolman-Oppenheimer-Volkoff (TOV) equation. In order to find $p(r)$ an equation of state of the form $p(r)=p(\rho(r))$ should be provided. One then gives the value zero for the central mass $(m(r=0)=0)$ so that there are no singularities beforehand and some adequate value for the central pressure. At the point along the radial direction where $p=0$ one finds the surface of the star, with radius $R$, and with a given total mass $M$. This solution can then be joined into the correct exterior vacuum solution, the Schwarzschild solution, giving a full description of the star's spacetime. For a consistent procedure see, e.g., [8, 9]. For an explanation of the possible different equations of state see [10] (see also [11] which in addition gives a general review on relativistic star structure). A simple equation of state is the polytropic one $p=\omega \rho^{\gamma}$, where $\omega$ is a constant and $\gamma$ is the polytropic exponent, as proposed by Tooper [12] in a general relativity setting, who also found solutions for general relativistic compact stars. For instance the interior Schwarzschild solution has $\gamma \rightarrow \infty$ and so the equation of state for the matter is $\rho=$ constant, with the pressure $p(r)$ adjusting itself to yield a static configuration. In [9] the TOV method is used to find the exact interior Schwarzschild solution by imposing the equation of state $\rho=$ constant in the TOV equation.

\subsubsection{The Buchdahl limit}

There is also an interesting and important limit in the theory of compact stars. It is the Buchdahl limit [13], and it is a limit of limits. The Buchdahl limit establishes that for a perfect fluid sphere of radius $R$ and mass $M$, if $R \geq$ $\frac{9}{4} M$ (or $R \geq \frac{9}{8} r_{+}$, where $r_{+}=2 M$ is the gravitational radius) then there is no equilibrium solution whatsoever. It means that, independently of the equation of state, this limit is absolute. It can be found by searching for the condition that yields an infinite pressure at the star's center. For instance, the Schwarzschild interior solution, with constant energy density for the matter (the stiffest equation of state that one can imagine), is a concrete example of the Buchdahl inequality. Presumably, when the limit is violated, the object collapses into a black hole.

Note the difference between the Oppenheimer-Volkoff limit and the Buchdahl limit. The Oppenheimer-Volkoff limit operates on a set of perfect fluid configurations whose matter obeys a given equation of state, say $p=$ $\omega \rho^{\gamma}$, with $\omega$ and $\gamma$ fixed, but whose central energy density is increased from member to member of the set. It gives a maximum mass, and through the radius-mass relation, a minimum radius. For a neutron star the maximum mass is of the order of $M \simeq 3 M_{\odot}$ and the corresponding minimum radius is $R \simeq 1.7 r_{+}$. The Buchdahl limit operates on a set of perfect fluid configurations, whose members have the stiffest equation of state $\rho=$ constant, for any constant, and whose central pressure is increased from member to member of the set up to infinity. This yields the limit $R \geq \frac{9}{8} r_{+}=1.125 r_{+}$.

\subsubsection{In brief}

Compact object is a term used to refer to astrophysical objects whose nature is related to the existence of pressure degeneracy to sustain the object against gravitational collapse and have in common the feature that they are all small for their mass. White dwarfs [14], neutron stars [15], and black holes [16-18] are well documented and well known objects, see also [19].

Two important quantities in the study of compact objects are the Oppenheimer-Volkoff mass limit, and the Buchdahl radius to mass limit.

\subsection{Electric compact stars}

\subsubsection{Electric compact stars, the electric TOV equation and the electric Oppenheimer-Volkoff limit}

Given that compact objects exist, a natural question that can be made is what is the maximum compactness that such an object can stand. Matter fields of perfect fluid type with a stiff equation of state cannot get more compact than the Buchdahl limit. Is it the case that allowing for other kinds of fields or even modifying somehow the gravitational field into some extension of general relativity one can get frozen stars, i.e., stars as compact as their own gravitational radii?

The simplest known field that can be added to a given matter within the star is the electric field. By finding a generalized TOV equation [20], one can seek for solutions of electric charged compact objects. Imposing different equations of state some of these solutions have indeed been devised [21-26].

Among the several new properties of these electric compact objects it was found that the OppenheimerVolkoff limit, the mass limit that sets in when the gravitational field due to the pressure overcomes its own support, gets larger as the electric charge of the matter increases, with the radius of the limiting configuration also increasing, see e.g. [24]. 


\subsubsection{The electric Buchdahl limit}

The Buchdahl limit also gets modified when electric charged is added to the matter particles. For charged spheres, the analogous of the Buchdahl limit has been worked out first in [27]. A development was performed in [28] and in [29] where a sharp bound for the star's radius $R$ to mass $M$ quotient, $R / M$ is given, namely, $R / M \geq$ $\left(1 / 3+\sqrt{1 / 9+Q^{2} / 3 R^{2}}\right)^{-2}$. Here it was considered that the matter obeys the conditions $p+2 p_{T} \leq \rho, p \geq 0$ and $\rho \geq 0$, where $\rho, p$, and $p_{T}$ are the energy density, the radial pressure and the tangential pressure of the fluid, respectively. Interestingly, for $Q=M$ this result admits the extremal case $R=M$ solution. Since in this extremal case the horizon radius $r_{+}$is $r_{+}=M$, one finds that for a star satisfying the extremal condition $Q=M$ the Buchdahl limit is such that the radius of the star is as compact as the gravitational radius $R=r_{+}$.

This is a remarkable result in many ways. Are there star solutions that are as compact as their own gravitational radii? Certainly there are.

\subsubsection{Electric compact stars and other methods: The Buchdahl and the quasiblack hole limits}

The TOV equation is a synthetic method to encode and analyze the structure of compact stars. It is most useful when one needs to integrate Einstein equations numerically, but it is by no means the only method. In many instances Einstein equations can be simplified due to symmetries or coincidences which are not displayed when use of the TOV method is made. This is the case of some of the works considered in this subsection.

By using Majumdar-Papapetrou matter, i.e., pressureless matter in which the energy density and the electric charge density are equal in appropriate units, and joining it into an extremal Reissner-Nordström solution, Bonnor previously found that these objects could have a radius as near as one wishes to their own gravitational radius $[30,31]$ (see also [32]). These star configurations are held against collapse by electric repulsion. Such solutions were improved in [33] where $C^{\infty}$ charged matter also showed the transition to a new gravitational field state at the gravitational radius of the configuration. This new gravitational state of a compact star is called a quasiblack hole and is the realization of the Buchdahl limit found in $[27,29]$ for electric configurations. Quasiblack holes can also be considered the real frozen stars, an alternative previous name given to black holes [16].

The inclusion of pressure into the matter also enabled to find exact electric star solutions in which quasiblack holes, or frozen stars, appear as limiting configurations [34]. Mention should be made to the works [22, 23] where consideration of an incompressible fluid in the presence of electric charge, and using the TOV method, led to relativistic charged sphere solutions which taking the limit to the black hole regime is still a compact star, i.e., a quasi- black hole. Quasiblack holes also arise from Yang-MillsHiggs matter, Einstein-Cartan matter with spin and torsion, rotating disk matter systems, and simple shells of matter, see [35] for a review.

The properties of these very peculiar compact objects have been worked out in detail, e.g., in [36, 37], see also [38]. They are objects on the limit of becoming extremal black holes, but unlike a black hole, there is no collapse of the progenitor star and the properties of matter inside the star are relevant. The spacetime is regular throughout although falling observers experience infinite tidal forces and the inner and outer regions turn hermetic. For these compact objects one can find a mass formula and their entropy yields results that match those for pure black holes, see [35].

\subsubsection{Compact stars with fields different from electric and in alternative theories of gravity}

There are general relativistic compact objects other that neutron stars, black holes and quasiblack holes. An important class of such objects are the regular black holes, i.e., objects that have all the properties of black holes but do not show a singularity at their core. For an electric realization of such objects see [39].

Of course, fields other than Maxwell and charged matter can be put or added, giving rise to several different compact objects such as gravastars, boson stars and also regular black holes. In addition, theories of gravitation, different from general relativity, can be used giving rise to compact objects displaying their own peculiarities showing that the study of compact stars comprises one of the fundamental subjects in any gravitational theory, for a review see [40].

\subsection{This work}

Our aim here is to explore a particular class of spherically symmetric cold charged fluid spheres, i.e., cold charged stars. We investigate electrically charged polytropic spheres. Many features we have mentioned will appear. The compact stars that will appear in this study have an Oppenheimer-Volkoff limit, a Buchdahl limit and a quasiblack hole limit.

The present paper is structured as follows. To properly define the physical quantities of the model we write in Sect. 2 the Einstein-Maxwell equations for a charged perfect fluid. The explicit set of equations in the case of spherical symmetry for a static spacetime in Schwarzschild-like coordinates are given and put in the TOV form. To close the system, an equation of state of polytropic form and a charge density profile are defined. Section 3 is devoted to report the general properties of the spheres in terms of the polytropic exponent. The Oppenheimer-Volkoff limit is analyzed as well as other properties of the spheres, such as their radius to mass ratio. In taking an infinite polytropic exponent, i.e., a stiff 
equation of state, one can find with care the Buchdahl and quasiblack hole limits. A study of the dependence of the speed of sound as a function of the polytropic index is also performed. In Section 4 we conclude.

\section{BASIC EQUATIONS, THE EQUATIONS OF EQUILIBRIUM, AND THE EQUATIONS OF STATE}

\subsection{Basic equations}

For completeness we start by writing the EinsteinMaxwell equations in the presence of charged matter, $c=1=G$,

$$
\begin{aligned}
& G_{\mu \nu}=8 \pi T_{\mu \nu}, \\
& \nabla_{\nu} F^{\mu \nu}=4 \pi J^{\mu},
\end{aligned}
$$

where the Greek indices $\mu, \nu$, etc., run from 0 to 3 ; the Einstein tensor is $G_{\mu \nu}=R_{\mu \nu}-\frac{1}{2} g_{\mu \nu} R$, with $R_{\mu \nu}$ and $g_{\mu \nu}$ being respectively the Ricci and the metric tensors, and $R$ being the Ricci scalar. $T_{\mu \nu}$ stands for the energymomentum tensor, which, in the present study is written as $T_{\mu \nu}=M_{\mu \nu}+E_{\mu \nu} . M_{\mu \nu}$ stands for the energymomentum tensor of a perfect fluid that is given by

$$
M_{\mu \nu}=p g_{\mu \nu}+(p+\rho) U_{\mu} U_{\nu}
$$

where $\rho$ is the energy density, $p$ is the pressure, and $U_{\mu}$ is the fluid's four velocity. The choice of a perfect fluid implies that the flow of matter is adiabatic, no heat flow, radiation, or viscosity is present [8]. $E_{\mu \nu}$ is the electromagnetic energy-momentum tensor,

$$
4 \pi E_{\mu \nu}=F_{\mu}^{\gamma} F_{\nu \gamma}-\frac{1}{4} g_{\mu \nu} F_{\gamma \beta} F^{\gamma \beta}
$$

where the Faraday-Maxwell strength tensor is

$$
F_{\mu \nu}=\nabla_{\mu} A_{\nu}-\nabla_{\nu} A_{\mu}
$$

with $\nabla_{\mu}$ representing the covariant derivative, and $\mathcal{A}_{\mu}$ the electromagnetic gauge field. In addition, the electric current density is written as

$$
J^{\mu}=\rho_{e} U^{\mu},
$$

where $\rho_{e}$ is the electric charge density.

\subsection{The equations of equilibrium}

In order to describe a static fluid distribution with spherical symmetry, the line element is assumed to be of the following form

$$
d s^{2}=-B(r) d t^{2}+A(r) d r^{2}+r^{2} d \theta^{2}+r^{2} \sin ^{2} \theta d \phi^{2},
$$

where $(t, r, \theta, \phi)$ are the usual Schwarzschild-like coordinates, with the metric functions $A(r)$ and $B(r)$ depending on $r$ alone.
Imposing that there is a static spherically symmetric electric field implies that the only nonzero components of the Maxwell strength tensor is $F^{\mu \nu}$ are $F^{t r}=-F^{r t}$, with $F^{t r}$ being a function of the radial coordinate $r$ alone, the other terms of the Maxwell tensor are identically zero. Hence, the only non-vanishing component of Maxwell equations (2) is given by

$$
\frac{d q(r)}{d r}=4 \pi \rho_{e}(r) r^{2} \sqrt{A(r)}
$$

where $q(r)=r^{2} \sqrt{A(r) B(r)} F^{t r}(r)$ is the total electric charge inside a sphere of radial coordinate $r$, which does not depend on the timelike coordinate $t$.

In the present case, considering the metric (7), the nonzero components of the Einstein equations (1) are

$$
\begin{aligned}
& \frac{d}{d r}\left[r A^{-1}(r)\right]=1-8 \pi r^{2}\left[\rho(r)+\frac{q^{2}(r)}{8 \pi r^{4}}\right] \\
& \frac{r B^{-1}(r)}{A(r)} \frac{d B(r)}{d r}+\frac{1}{A(r)}=1+8 \pi r^{2}\left[p(r)-\frac{q^{2}(r)}{8 \pi r^{4}}\right]
\end{aligned}
$$

As usual, we define a new quantity $m(r)$ representing the mass inside the shell of radial coordinate $r$ in such a way that

$$
A^{-1}(r)=1-\frac{2 m(r)}{r}+\frac{q^{2}(r)}{r^{2}} .
$$

Now, replacing Eq. (11) into Eq. (9) it gives

$$
\frac{d m(r)}{d r}=4 \pi r^{2} \rho(r)+\frac{q(r)}{r} \frac{d q(r)}{d r},
$$

that represents the mass (energy) conservation, as measured in the matter's frame.

Moreover, from the Bianchi identities $\nabla_{\mu} T^{\mu \nu}=0$, it follows

$$
\frac{d B}{d r}=\frac{q B}{2 \pi r^{4}(p+\rho)}\left(\frac{d q}{d r}\right)-\frac{2 B}{p+\rho}\left(\frac{d p}{d r}\right),
$$

where, to shorten equations and simplify notation, we dropped the function dependence, $B(r)=B, q(r)=q$, and so on.

Finally, replacing Eq. (8) and the conservation equation (13) into Eq. (10) it yields

$$
\frac{d p}{d r}=-(p+\rho) \frac{\left(4 \pi r p+\frac{m}{r^{2}}-\frac{q^{2}}{r^{3}}\right)}{\left(1-\frac{2 m}{r}+\frac{q^{2}}{r^{2}}\right)}+\rho_{e} \sqrt{A} \frac{q}{r^{2}},
$$

which is the Tolman-Oppenheimer-Volkoff (TOV) equation $[4,5]$ modified to the study of equilibrium of an electrically charged fluid, see [20] (see also [24]).

It is clear that equations (8), (11), (12) and (14) are not enough to solve for the six variables $q(r), A(r), m(r)$, $\rho(r), p(r)$ and $\rho_{e}(r)$, since there are two degrees of freedom. The two missing equations (or constraints) are generally obtained from a model of matter, in this case, of 
the charged fluid. To complete this set of equations, it is commonly considered an equation of state relating the pressure with the energy density of the fluid. Moreover, for the electrically charged fluid, it is also needed a relation defining the electric charge density, see below. The resulting set of equations constitute the complete set of structure equations which, with some appropriate boundary conditions, can be solved simultaneously.

The final step to set up the system is defining the boundary conditions for the sought solutions. In the present case, those are chosen at $r=0$, the center of the sphere, and are: $m(r=0)=0, q(r=0)=0$, $p(r=0)=p_{c}, \rho(r=0)=\rho_{c}, \rho_{e}(r=0)=\rho_{e c}$. These conditions imply in $A(r=0)=1$. The surface of the star $r=R$ is found when $p(R)=0$. For the numerical calculations, the inputs in the system of equations are the central energy density $\rho_{c}$ which through the equation of state yield the central pressure $p_{c}$, and the central charge density $\rho_{e c}$.

The metric exterior to the sphere is given by ReissnerNordström metric

$d s^{2}=-\left(1-\frac{2 M}{r}+\frac{Q^{2}}{r^{2}}\right) d T^{2}+\frac{d r^{2}}{1-\frac{2 M}{r}+\frac{Q^{2}}{r^{2}}}+r^{2} d \Omega^{2}$,

with $M$ and $Q$ being respectively the total mass and the total charge of the sphere. The time $T$ is proportional to the inner time $t$ and the radial coordinate $r$ is identical to the radial coordinate of the interior region. The full set of boundary conditions at the surface of the star is $B(R)=$ $1 / A(R)=1-2 M / R+Q^{2} / R^{2}, m(R)=M, q(R)=$ $Q$, besides $p(R)=0$, and so $\rho(R)=0$ for a polytropic equation of state, which, together with a smooth charge density profile, is equivalent to the full set of junction conditions for boundary layers in general relativity in this particular instance.

\subsection{Equation of state and charge density relation}

\subsubsection{The equation of state}

As mentioned above, to complete the set of equations it is necessary to add two more relations to the charged fluid system. Usually, an appropriate equation of state is furnished. Among the simplest choices, a polytropic equation of state is frequently used. For the purpose of the present analysis, following Tooper [12], it is convenient to choose the following polytropic relation

$$
p=\omega \rho^{\gamma},
$$

where $\omega$ is the polytropic constant and we use the name polytropic exponent for the parameter $\gamma$.

Equation (16) is a good equation of state for a Newtonian ideal fluid, indeed it is equivalent to that of a nonrelativistic fluid for small pressures, when compared to the energy density [10]. However, for high densities and pressures, Eq. (16) violates causality conditions for any $\gamma$. To see that we consider the speed of sound $c_{s}$ within the fluid. The square of the sound speed is $c_{s}^{2}=d p / d \rho$ and so the equation of state (16) gives $c_{s}^{2}=\gamma p / \rho$. On the basis of this equality, we can determinate the value of the speed of sound across a given star as a function of the radial coordinate $r$. Moreover, we can see that for sufficiently large pressure the speed of sound becomes larger than the speed of light. In considering large values of $\gamma$ here we will find high pressures at the center violating causality and the usual energy conditions. Even though this is a drawback, as we will see it is interesting to analyze the regime of large polytropic exponents and check the properties of the corresponding polytropic spheres. With this models in hand we can then compare with other models of charged stars in the literature. We can also verify how the speed of sound depends on the polytropic exponent, central pressure, charge fraction and other physical quantities of the model. Furthermore, in investigating the dependence of the structure of the charged spheres on a gamut of different polytropic exponents one can take the limit of very large $\gamma$ when the fluid becomes incompressible and situations similar to the Schwarzschild interior solution appear. More specifically, as we shall see bellow, in the case of uncharged or charged spheres the Buchdahl limit is approached for $\gamma \rightarrow \infty$. In addition, for very large $\gamma$ the quasiblack hole limit is reached.

\subsubsection{The charge density relation}

In order to study the effects of the electric charge in the structure of polytropic charged spheres we need also to define the charge density profile. For simplicity, we assume that the charge density is proportional to the energy density, see [24] (see also [21, 26]),

$$
\rho_{e}=\alpha \rho
$$

where $\alpha$ is the charge fraction and, in geometric units, is a dimensionless constant.

As already mentioned, the effect of the electric charge in the structure of the stars has been studied in previous works considering several values of the charge fraction $\alpha$ for a fixed equation of state, i.e., by taking Eq. (16) with fixed $\gamma=5 / 3$ [24]. In the present work we are interested in studying all possible compact star solutions including their extreme limits, such as quasiblack hole solutions. More specifically, using the equation state (16), we consider different charge amounts $\alpha$ and also different polytropic exponents $\gamma$. Therefore, given the value of the charge fraction $\alpha$, the polytropic constant $\omega$, and the central energy density (or the central pressure), we can analyze the system as a function of $\gamma$. 


\section{THE STRUCTURE OF POLYTROPIC CHARGED STARS WITH VARYING $\gamma, 0<\gamma<\infty$}

\subsection{General remarks}

We now present the structure of charged spheres made up of polytropic fluids for various values of the polytropic exponent $\gamma$ and charge fraction $\alpha$. We investigate the type of compact objects that might arise from these solutions, and whether they have a quasiblack hole limit. In the analysis we have found that such a regime is approached in the limit $\gamma \rightarrow \infty$. In order to find the whole spectrum of objects and in particular the quasiblack hole, we integrate the system of equations considering many different values of the polytropic exponent $\gamma$. For the numerical integration we rewrite equations (8), (11), (12), (14), (16), and (17), as well as the boundary conditions adopted in the center of the star, in a dimensionless form (see Appendix A). The relevant equations then take the form given by Eqs. (A5), (A6), and (A7). For each given value of $\gamma, \alpha$, and $\rho_{c}$ (or, equivalently, $p_{c}$ ), the resulting system of equations is numerically solved through a fourth order Runge-Kutta method. The main results are displayed now.

Since we analyze the limit o large polytropic exponents, we plot the results in terms of $\tanh \gamma$, for $\gamma$ in the interval $[4 / 3,17.1]$. Hence, it is interesting to show explicitly the values of $\tanh \gamma$ as a function of $\gamma$. This is done in Table I.

\begin{tabular}{|c|c|c|c|c|c|c|c|}
$\gamma$ & $4 / 3$ & $5 / 3$ & 2.0 & 3.0 & 5 & 17.1 & $\infty$ \\
\hline $\tanh \gamma$ & 0.8701 & 0.9311 & 0.9640 & 0.9950 & 0.9999 & 1.000 & 1.000
\end{tabular}

TABLE I: The hyperbolic tangent, $\tanh \gamma$, of the polytropic exponent $\gamma$.

In presenting the numerical results we continue to put $c=1$ but $G$ is given in MKS units, $G=6.67384 \times$ $10^{-11} \mathrm{~m}^{3} / \mathrm{kg} \mathrm{s}^{2}$.

\subsection{Numerical input values}

For a generic equation of state as (16) in a star, besides the polytropic exponent $\gamma$, there are two free parameters, the polytropic constant $\omega$ and the central energy density $\rho_{c}$. On the other hand, in order to analyze the structure of charged polytropic spheres for large values of the polytropic exponent one must be careful when choosing the normalization factors for the numerical calculation, because the convergence strongly depends on such a choice. Hence, for convenience, and also for the sake of comparison with the results in the literature, the central energy density of a standard neutron star model, that is, $\rho_{c} \sim 10^{17} \mathrm{~kg} / \mathrm{m}^{3}$ (see, e.g., [15]), is a good reference value for our numerical analysis. With this in mind and recalling that the equation of state (16) is used, following [24] (see also Appendix A) a value of $\omega$ is picked up in order that for $\gamma=5 / 3$ and a suitably chosen cen- tral energy density, the solution is close to the values for real neutron stars. In fact, it is convenient to normalize the polytropic constant $\omega$ in such a way that it turns out a function of the polytropic exponent $\gamma$, namely, $\omega=\omega(\gamma)=1.47518 \times 10^{-3}\left(1.78266 \times 10^{15} \mathrm{~kg} / \mathrm{m}^{3}\right)^{1-\gamma}$. With such a choice, a natural normalization factor for the numerical calculations related to the TOV equation would be $\rho_{0}=1.78266 \times 10^{15} \mathrm{~kg} / \mathrm{m}^{3}$, corresponding to the pressure $p_{0}=2.62974 \times 10^{12} \mathrm{~kg} / \mathrm{m}^{3}$, which is independent of $\gamma$. However, since we want to verify the limit of arbitrarily large polytropic exponents (see below), it is necessary to consider a normalization factor $\rho_{0}$ not larger than the central energy densities of interest. We then have chosen to normalize quantities in terms of the central energy density (see Appendix A).

We have made calculations for many different values of the polytropic exponent $\gamma$, the charge fraction $\alpha$, and the central energy density $\rho_{c}$. We are mostly interested here in effects of varying the exponent $\gamma$ in order to find, for high values of $\gamma$, the quasiblack hole limit. We then fix the central energy density and vary $\gamma$ starting from the value $\gamma=4 / 3$ which corresponds to the usual relativistic ideal fluid. Note that for large $\gamma$ the central pressure becomes very large, see the equation of state (16).

We show below the results obtained for different central energy densities and some different values of the polytropic exponents. Since one of the goals is the search for quasiblack holes, we have chosen a central energy density ten times larger than the normalization factor, $\rho_{c}=10 \rho_{0}=1.78266 \times 10^{16} \mathrm{~kg} / \mathrm{m}^{3}$. For this value of central energy density, the maximum value of the polytropic exponent that produces good results is approximately 17.0667 (17.1, for short). For larger values, the convergence is slow and the results present numerical instabilities. In the case $\gamma=17.1$ we have that the central pressure is about 15.8 orders of magnitude larger than the $\gamma=4 / 3$ central pressure, i.e., $p_{c}^{(\gamma=17.1)} / p_{c}^{(\gamma=4 / 3)}=$ $\omega^{(\gamma=17.1)} \rho_{c}^{17.1} / \omega^{(\gamma=4 / 3)} \rho_{c}^{4 / 3} \simeq 1.0 \times 10^{15.8}$. Since $p_{c}^{(\gamma=17.1)}$ is much larger than $p_{c}^{(\gamma=4 / 3)}$, in the numerical analysis we consider the first value as satisfying the condition for the limit of arbitrarily large pressure, we considered it as an infinite central pressure.

As usual, the values of the mass $M$, charge $Q$ and radius $R$ of the star are found when the pressure at the surface of the star is equal to zero $p(r=R)=0$. Since this rarely is the case, the numerical code is stopped when the pressure becomes smaller than a appropriately chosen very small value, or when it changes sign from positive to negative values.

\subsection{Radius of the spheres as a function of the mass for fixed polytropic exponent and different charge fractions: The Oppenheimer-Volkoff limit}

In Figs. 1 and 2 we plot the radius of the resulting spheres as a function of the mass, normalized to the Sun's mass $M_{\odot}$, for $\gamma=4 / 3$ and $\gamma=5 / 3$, respectively, and for a few values of the charge fraction $\alpha$. The exponent 
$\gamma=4 / 3$ represents soft relativistic matter. Exponents like $\gamma=5 / 3$ or higher represent harder matter cores. The considered central energy densities $\rho_{c}$ are in the interval $1.0 \times 10^{13}$ to $1.0 \times 10^{20} \mathrm{~kg} / \mathrm{m}^{3}$. These graphs are to be compared to the results of Ref. [24], where $\gamma=5 / 3$ fixed and the maximum value of $\alpha$ is of about 0.96 , while here we show results for $\alpha$ up to 0.99. For a given charge fraction, the usual behavior of the polytropic cold stars is noticed, with the radius of the star decreasing with the mass as the central energy density grows. Also, the spiraling behavior of the curve $R \times M$ is observed for high central densities (see, e.g., [17]).

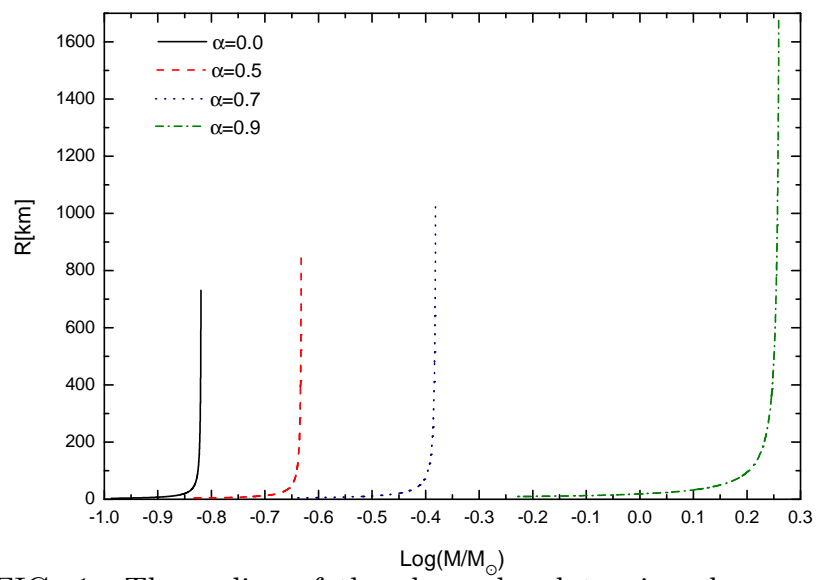

FIG. 1: The radius of the charged polytropic sphere as a function of the mass for $\gamma=4 / 3$ and a few values of charge fraction $\alpha$. The considered central energy densities are in the interval $1.0 \times 10^{13}$ to $1.0 \times 10^{20} \mathrm{~kg} / \mathrm{m}^{3}$. The Chandrasekhar and Oppenheimer-Volkoff mass limits coincide in this $\gamma=4 / 3$ case.

In Fig. 1 for $\gamma=4 / 3$, one can see the Chandrasekhar mass limit appearing distinctly in the vertical lines of the plots together with the Oppenheimer-Volkoff limit, the points where the vertical lines turn to the left. Clearly, the two mass limits coincide in this $\gamma=4 / 3$ instance, as perhaps could be expected following the heuristic arguments given in [6]. Nonetheless it would be worth exploring this coincidence. In addition to a mass limit, the Oppenheimer-Volkoff limit also gives a minimum radius for the star (the Chandrasekhar minimum radius is zero, as it uses Newtonian gravitation rather than general relativity).

In Fig. 2 for $\gamma=5 / 3$, one can see the OppenheimerVolkoff limit, the points where the inclined lines inflect to the left. In this case, as for all $\gamma \neq 4 / 3$ cases, there is no Chandrasekhar limit.

\subsection{Properties of the spheres as a function of the polytropic exponent: Towards the Buchdahl limit}

\subsubsection{Radius of the spheres as a function of the polytropic exponent}

The dependence of the curve $R \times M$ on the polytropic exponent can be seen in Fig. 3, where we plot the ra-

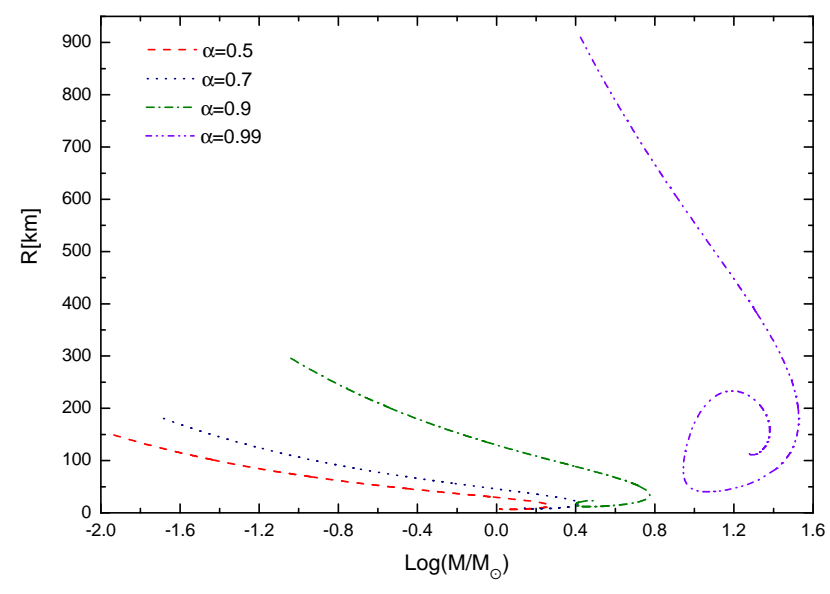

FIG. 2: The radius of the charged polytropic sphere as a function of the mass for $\gamma=5 / 3$ and a few values of charge fraction $\alpha$. The considered central energy densities are in the interval $1.0 \times 10^{13}$ to $1.0 \times 10^{20} \mathrm{~kg} / \mathrm{m}^{3}$. The Oppenheimer-Volkoff mass limit appears clearly, and there is no Chandrasekhar limit for $\gamma \neq 4 / 3$.

dius of the resulting spheres as a function of the mass for $\alpha=0.5$ and a few values of the polytropic exponent $\gamma$. As in the case of Fig. 2, the considered central energy densities are in the interval $1.0 \times 10^{13}$ to $1.0 \times 10^{20} \mathrm{~kg} / \mathrm{m}^{3}$. These graphs show results completely new and we do not find similar analysis in the literature for comparison. Namely, as the polytropic exponent increases, the inclination of the curve $R \times M$ decreases becoming approximately horizontal for $\gamma$ around 2.0. This can be understood by taking into account that a very large polytropic exponent implies an approximately constant energy density, $\rho=\rho_{0}$, resulting in a relation of the form $M \sim 4 \pi \rho_{0} R^{3} / 3$, which means that the radius of the sphere increases with the mass and also with the central energy density. It is interesting to say that the general behavior of the curves for large $\gamma$ is independent of $\alpha$, namely the dominant behavior of negative $d R / d M<0$ for small $\gamma$ (typically for $\gamma<2.0$ ) changes to $d R / d M>0$ for large $\gamma$.

We plot the radius of the resulting spheres as a function of the mass for $\gamma=17.0667$ and a few values of the charge fraction $\alpha$ in Fig. 4. Notice that the charge fraction does not change significantly the results. Since for large values of $\gamma$ the central pressure becomes very large, the numerical calculations can not be performed for large values of the central energy density, so that in this case the considered values of $\rho_{c}$ are in the interval $2.0 \times 10^{15}$ to $2.4 \times 10^{16} \mathrm{~kg} / \mathrm{m}^{3}$.

\subsubsection{Mass of the spheres as a function of the polytropic} exponent

Fig. 5 shows the total gravitational mass of the star as a function of the polytropic exponent (in logarithmic scale) for the central density $\rho_{c}=1.78266 \times 10^{16} \mathrm{~kg} / \mathrm{m}^{3}$ 

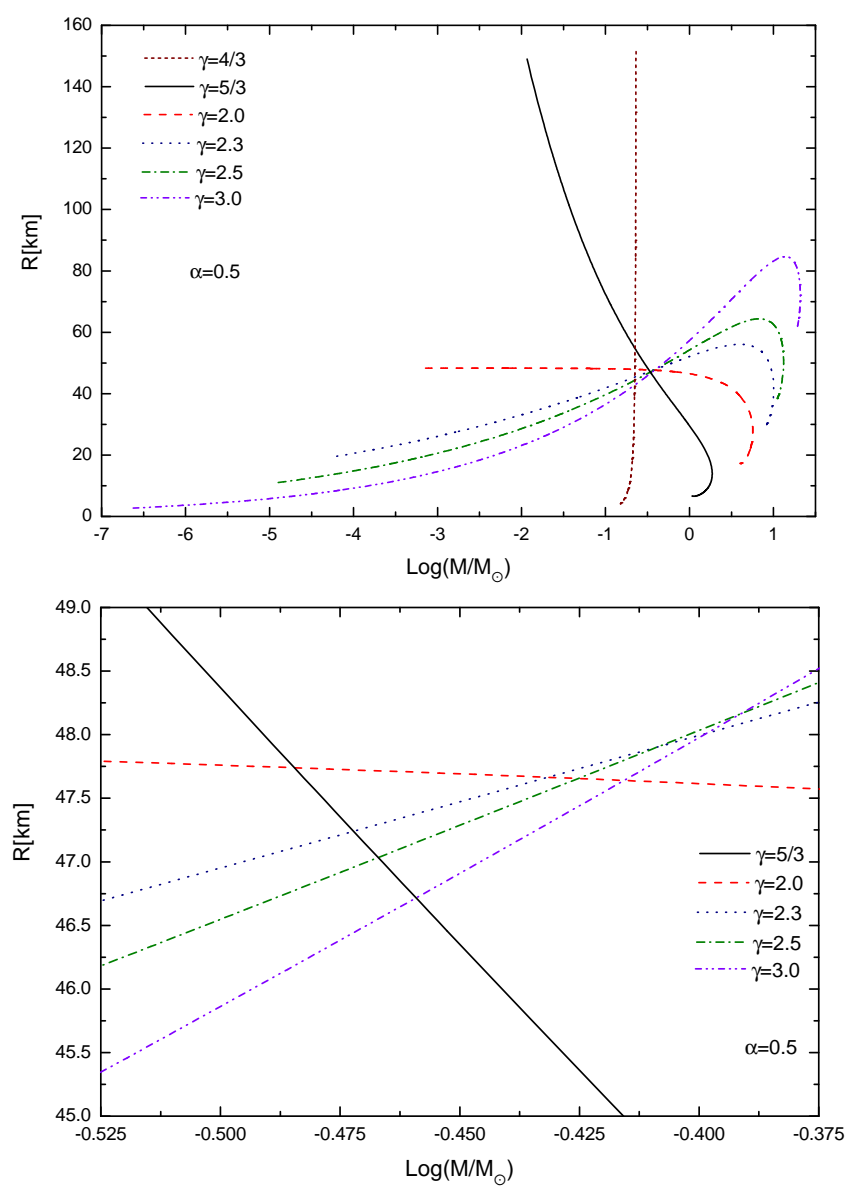

FIG. 3: Top: The radius of the charged polytropic sphere as a function of the mass for $\alpha=0.5$ and a few values of the polytropic exponent $\gamma$. The considered central energy densities are in the interval $1.0 \times 10^{13}$ to $1.0 \times 10^{20} \mathrm{~kg} / \mathrm{m}^{3}$. The curve for $\gamma=4 / 3$ was interrupted at the central energy density $\rho_{c}=1.72 \times 10^{15} \mathrm{~kg} / \mathrm{m}^{3}$, the complete behavior of this curve can be seen in the Fig. 1. Bottom: Amplification of the region where the lines intersect. Note the lines do not intersect at a point as could be inferred from the top panel. The line $\gamma=4 / 3$ is to the left of this bottom panel and does not appear.

and for several values of the charge fraction $\alpha$. The values of $\gamma$ considered are in the interval $4 / 3 \leq \gamma \leq 17.0667$. As one may observe, the gravitational mass increases with $\gamma$ in a rate that is larger for small charge fractions. For instance, in the case $\alpha=0.5$, the mass for $\gamma=4 / 3$ is approximately $M=0.23 M_{\odot}$ while for $\gamma=17.1$ it is $M=75.0 M_{\odot}$. That is to say, the mass grows about $32,509 \%$. On the other hand, for $\alpha=0.99$ the mass grows approximately $588 \%$. The growth of the mass with $\gamma$ may be understood by noticing that the central pressure increases with $\gamma$, so that the weight of more mass is supported against collapse.

The dependence of the mass with $\alpha$ can also be seen in Fig. 5. It increases monotonically with the charge fraction, and strongly depends on $\gamma$ too. In fact, for $\gamma=4 / 3$, the mass of the star increases about $11,451 \%$, from $M=0.23 M_{\odot}$ at $\alpha=0.5$ to $M=26.2 M_{\odot}$ at $\alpha=$ 0.99. In turn, for $\gamma=17.1$ and in the same interval

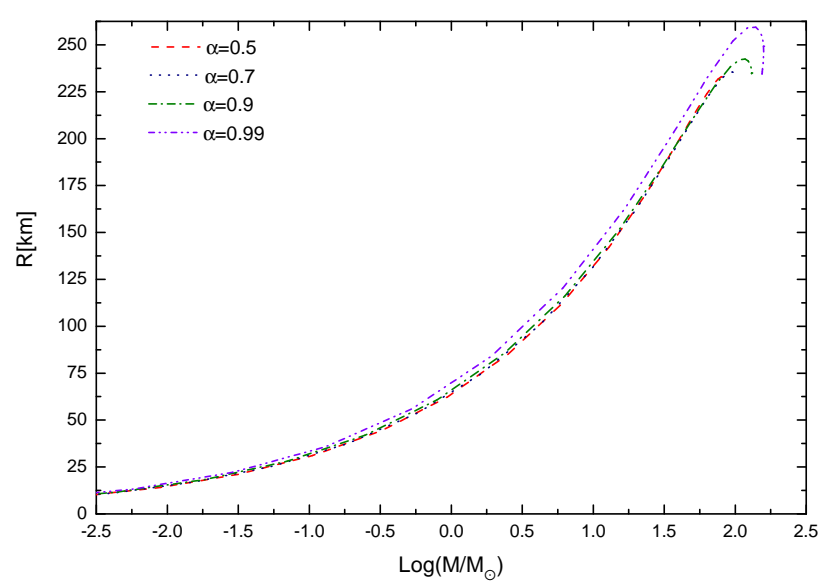

FIG. 4: The radius of the charged polytropic sphere as a function of the mass for $\gamma=17.0667$ and a few values of charge fraction $\alpha$. The considered central energy densities are in the interval $2.0 \times 10^{15}$ to $2.4 \times 10^{16} \mathrm{~kg} / \mathrm{m}^{3}$.

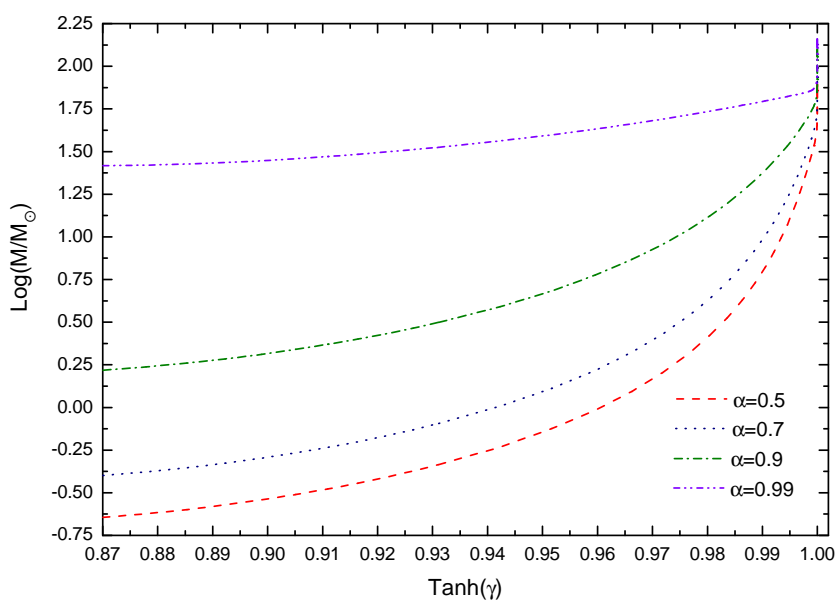

FIG. 5: The mass of the charged polytropic sphere as a function of the polytropic exponent considering the central energy density $\rho_{c}=1.78266 \times 10^{16} \mathrm{~kg} / \mathrm{m}^{3}$ and a few values of charge fraction $\alpha$.

of $\alpha$ the mass varies approximately $85.57 \%$, from $M=$ $82.8 M_{\odot}$ to $M=153.65 M_{\odot}$. This is shown explicitly in Fig. 6. Such a behavior is also easily explained since the electric charge acts as an effective pressure, helping the hydrodynamic pressure to support collapse. Hence, the two effects of increasing $\alpha$ and $\gamma$ act favorable to yield equilibrium configurations with large masses.

The value $\alpha=0.99$ is the maximum value of the charge fraction we could find equilibrium solutions in our numerical calculations for the largest value of $\gamma$ we considered. However, for $\gamma$ close to $5 / 3$ we can get results even for $\alpha=0.9999$ corresponding to spheres with very large masses and radii. 


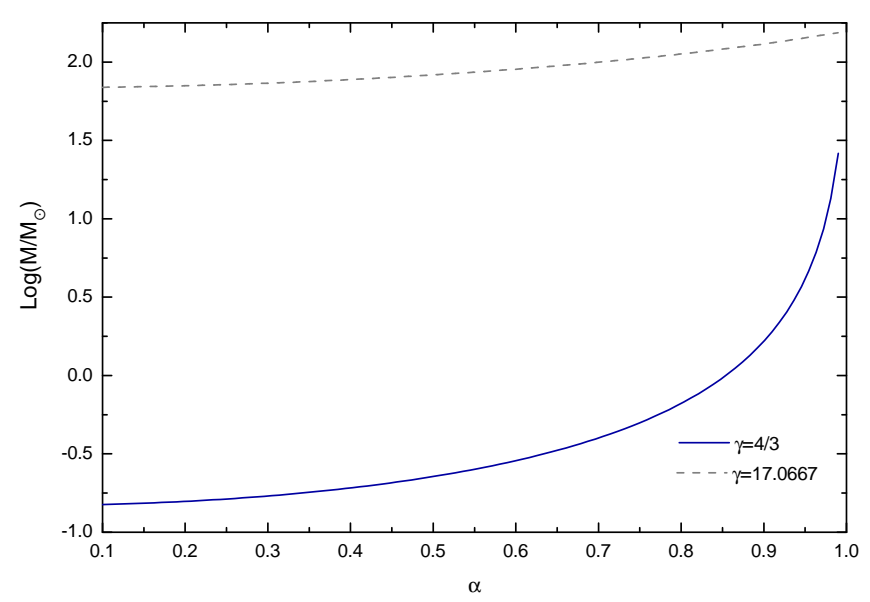

FIG. 6: The mass of the charged polytropic sphere as a function of the charge fraction $\alpha$ considering the central energy density $\rho_{c}=1.78266 \times 10^{16} \mathrm{~kg} / \mathrm{m}^{3}$ and two values of $\gamma$.

\subsubsection{The radius to mass ratio of the sphere as a function of the polytropic exponent}

The values of the ratio $R / M$ as a function of the polytropic exponent are shown in Fig. 7 in logarithmic scale. In this figure, as in Fig. 5, it is considered the central energy density $\rho_{c}=1.78266 \times 10^{16} \mathrm{~kg} / \mathrm{m}^{3}$ and a few different values of the charge fraction $\alpha$. The main feature to note is that $R / M$ decreases with increasing $\gamma$, a common behavior for all values of central energy densities we have investigated (even for those cases not showed in the figure). Note that in the case without charge, $\alpha=0.0$, and large polytropic exponent, $\gamma=17.1$, it is found $R / M \simeq 2.27$. If one extrapolates to $\gamma \rightarrow \infty$, giving the Schwarzschild interior solution, one finds the Buchdahl limit, $R / M=9 / 4=2.25$, as expected. This is shown more clearly in Fig. 8 (see also Sect. 3.5.2). On the other hand, for $\alpha=0.99$ and $\gamma=17.1$ the ratio $R / M$ is very close to unity, see the discussion below.

It is seen that for charged stars $(\alpha \neq 0.0)$ we obtain solutions that violate the original (uncharged) Buchdahl limit [13], i.e., we find $R / M<9 / 4$. Also, there are values of $R / M$ that are smaller than the bound for charged objects established in [28], which takes into account the contributions of the electric charge. For instance, in the case $\alpha=0.99$ and $\gamma \simeq 17.1$, we find $R / M \approx 1.03$, while the minimum value of $R / M$ calculated from the results obtained in [28], with our values for $M$ and $R$, is $R / M \gtrsim 3.18$. A possible explanation for such a different result is that our equation of state for large $\gamma$ implies arbitrarily large values of the central pressure, while in [28] it is assumed that the physical quantities are well behaved and finite.

As seen here, the combined conditions of large charge fraction and high polytropic exponent implies matter can be compressed beyond the original Buchdahl limit, up to the quasiblack hole limit, see below.

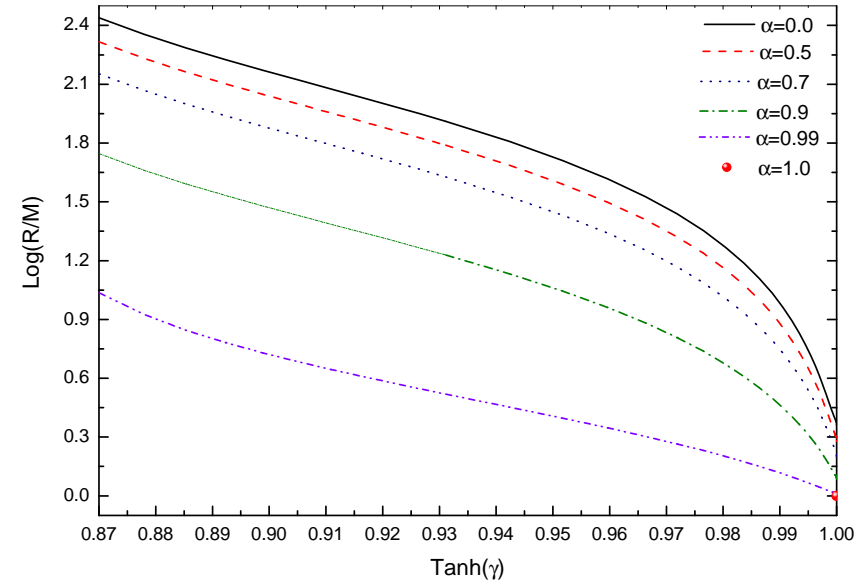

FIG. 7: Values of the ratio $R / M$ against the polytropic exponent $\gamma$ for a few values of the charge fraction $\alpha$. For large $\gamma$, the curves for $\alpha=0.0$ and for $\alpha=0.99$, approach the Buchdahl limit $R / M=9 / 4$ and $R / M=1$, respectively.

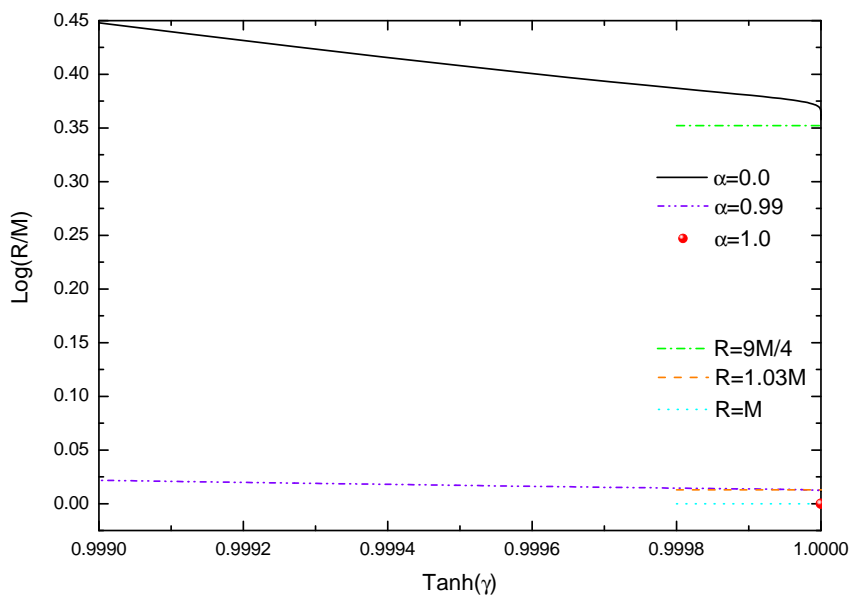

FIG. 8: The ratio $R / M$ as a function of the polytropic exponent for $\rho_{c}=1.78266 \times 10^{16} \mathrm{~kg} / \mathrm{m}^{3}, \alpha=0.0$, and $\alpha=0.99$, showing the extreme limit in each case. For $\alpha=0$ we get the Buchdhal limit, for $\alpha=1$ we get the quasiblack hole limit.

\subsubsection{The charge of the sphere as a function of the polytropic exponent}

The behavior of the charge of the sphere as a function of the polytropic exponent can be seen in Fig. 9, where we plot the ratio $Q / M$ versus the polytropic exponent in logarithmic scale for the same central density, $\rho_{c}=1.78266 \times 10^{16} \mathrm{~kg} / \mathrm{m}^{3}$, as in Fig. 7 , and for different values of charge fraction $\alpha$ as indicated. It is seen that $Q / M$ increases with the increasing of the polytropic exponent $\gamma$ for all $\alpha$. Moreover, the largest value $Q / M \approx 0.999793$ is found for the charge fraction $\alpha=0.99$ and $\gamma=17.0667$. These are the largest values our numerical code yield results without running into convergence troubles. For intermediate values of $\alpha$, the results show an unexpected dependence of the total amount of charge upon the polytropic exponent. For a 
given charge fraction $\alpha$, the total charge jumps from approximately $\alpha M$ for $\gamma \lesssim 2.0$ to approximately $1.3 \alpha M$ for $\gamma \gtrsim 4.0$. A possible interpretation of this result is that fluid spheres made of stiff matter (large $d p / d \rho$, see bellow) admit more charge than those made of soft matter (small $d p / d \rho)$.

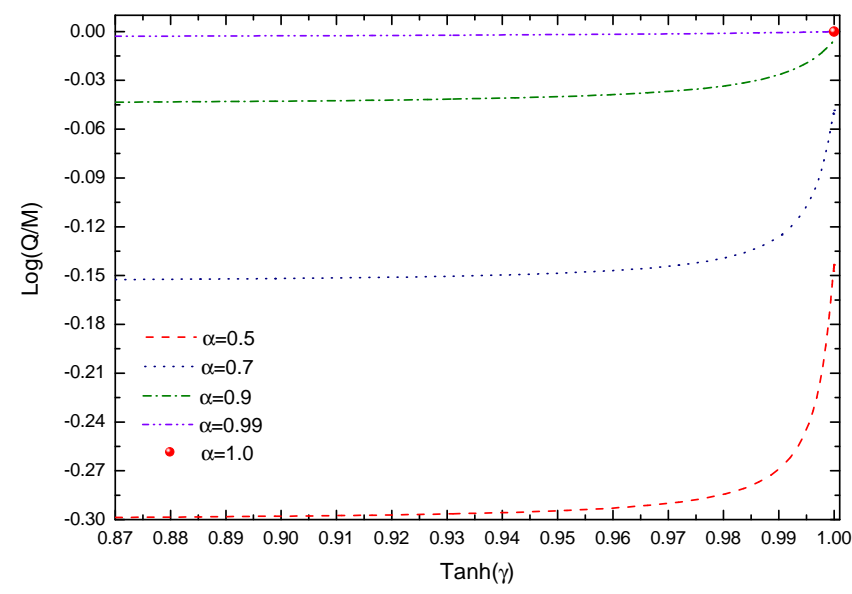

FIG. 9: Values of the ratio $Q / M$ as a function of the polytropic exponent for different charge fractions $\alpha$ and central energy density $\rho_{c}=1.78266 \times 10^{16} \mathrm{~kg} / \mathrm{m}^{3}$.

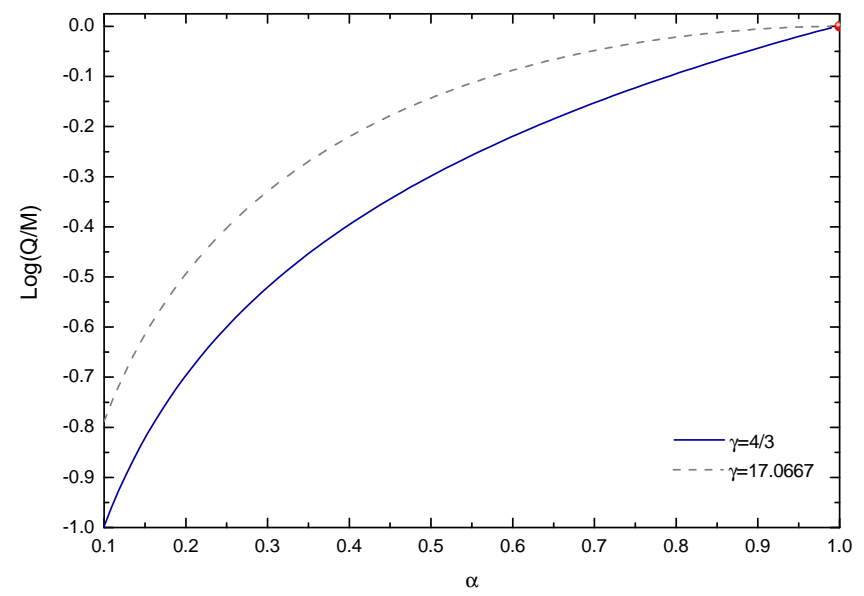

FIG. 10: Values of the ratio $Q / M$ as a function of the charge fraction for the polytropic exponent $\gamma=4 / 3$ and $\gamma=17.0667$ and central energy density $\rho_{c}=1.78266 \times 10^{16} \mathrm{~kg} / \mathrm{m}^{3}$. The curves for all $\gamma$ in the interval $[4 / 3,17.0667]$ are in between the two shown curves.

The dependence of the ratio $Q / M$ as a function of the charge fraction $\alpha$ for some values of the polytropic exponent $\gamma$ is shown in Fig. 10.

\subsection{The infinite polytropic exponent limit and the Buchdahl and quasiblack hole limits}

\subsubsection{Initial remarks}

The numerical analysis we have performed indicates that the quasiblack hole limit is not reached by polytropic charged fluid spheres, using the polytropic equation of state (16), with the polytropic exponent in the interval $4 / 3 \leq \gamma \leq 2.0$, and with smooth boundary conditions, i.e., with finite central pressure and zero pressure at the surface of the sphere.

However, previous results, and also some preliminary numerical calculations using the present formulation, suggest that the quasiblack hole limit can be found considering matter with equation of state other than the polytropic one. For instance, in the case of $\rho(r)=$ constant $[22,23]$ or constant total energy density [34], quasiblack holes were modelled by charged matter. Moreover, in the present framework we may get constant energy density in the limit of very large polytropic exponents.

In order to investigate that limit normalized quantities are necessary, as usual in numerical calculations. We call $p_{0}$ a particularly chosen value of the central pressure and considered it as the normalization factor for the pressure. Hence, using Eq. (16) to write $p_{0}=w \rho_{0}^{\gamma}$, we get

$$
\lim _{\gamma \rightarrow \infty} \frac{p}{p_{0}}=\lim _{\gamma \rightarrow \infty}\left(\frac{\rho}{\rho_{0}}\right)^{\gamma}=\left\{\begin{array}{l}
\infty, \quad \text { if } \quad \rho>\rho_{0} \\
0, \quad \text { if } \quad \rho<\rho_{0}
\end{array}\right.
$$

and

$$
\lim _{\gamma \rightarrow \infty} \frac{\rho}{\rho_{0}}=\lim _{\gamma \rightarrow \infty}\left(\frac{p}{p_{0}}\right)^{1 / \gamma}=1 .
$$

The same holds for the charge density in case one uses Eq. (17),

$$
\lim _{\gamma \rightarrow \infty} \frac{\rho_{e}}{\rho_{0 e}}=\lim _{\gamma \rightarrow \infty} \frac{\rho}{\rho_{0}}=\lim _{\gamma \rightarrow \infty}\left(\frac{p}{p_{0}}\right)^{1 / \gamma}=1 .
$$

Therefore, if the central energy density $\rho_{c}$ is larger than the normalization value $\rho_{0}$, the central pressure of such a sphere is infinite. On the other hand, if the central energy density is smaller than $\rho_{0}$, the central pressure vanishes and then no equilibrium solution is found. Moreover, in such a limit, independently of the value of $p_{c}$, i.e., independently of $\rho_{c}$, the energy density $\rho$ is a constant throughout the charged fluid sphere. This is the analogous of the Schwarzschild star, whose compactness is bounded by the Buchdahl limit.

Fig. 11 shows the relations $\rho(r) / \rho_{0}$ and $p(r) / p_{0}$ in terms of the radial coordinate for the charge fraction $\alpha=0.99$, polytropic exponent $\gamma=17.0667$ and central energy density $\rho_{c}=1.78266 \times 10^{16} \mathrm{~kg} / \mathrm{m}^{3}$. As it can be noted, the pressure inside the sphere decreases very fast with radius. Starting with a very high value at $r=0$, the pressure reaches its minimum value, ideally 


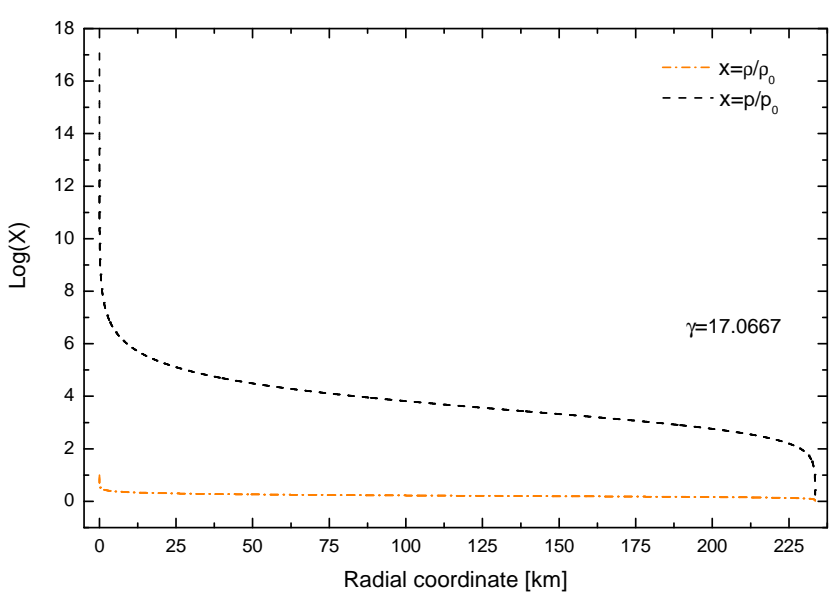

FIG. 11: The ratios $\rho(r) / \rho_{0}$ and $p(r) / p_{0}$ versus the radial coordinate for the central energy density $\rho_{c}=1.78266 \times$ $10^{16} \mathrm{~kg} / \mathrm{m}^{3}$, polytropic exponent $\gamma=17.0667$ and charge fraction $\alpha=0.99$ The normalization factors used are $\rho_{0}=$ $1.78266 \times 10^{15} \mathrm{~kg} / \mathrm{m}^{3}$ and $p_{0}=2.62974 \times 10^{12} \mathrm{~kg} / \mathrm{m}^{3}$.

equal to zero, at a value $R$ of the radial coordinate, which is identified as the radius of the charged sphere. Following the standard procedure, the numerical calculation is then stopped at that point, $r=R$, and the interior solution is matched to the exterior Reissner-Nordström solution. If the calculation is continued, the pressure would reach negative values and we discard those solutions. On the other hand, the energy density varies very slowly with the radial coordinate, starting with $\rho / \rho_{0}=10$ at $r=0$ and decreasing only one order of magnitude until very close to the surface of the sphere. In comparison to the pressure variation, the energy density is approximately a constant throughout the sphere. It is important to mention that the initial values of $\log \left(p / p_{0}\right)$ and $\log \left(\rho / \rho_{0}\right)$ differ by 16 (sixteen), and that due to this the behavior of the curve $\rho(r) / \rho_{0}$ is not fully clearly seen in Fig. 11 .

With total confidence, we may then extrapolate these results and state that our numerical analysis confirms the results for infinitely large polytropic exponents, as shown by Eqs. (18) and (19). In such a limit the charged sphere is similar to the Schwarzschild star in the sense that it has a constant energy density. That peculiarity in the energy density of the star, such as $\rho(r)=$ constant like in $[22,23]$ or total energy density equals a constant like in [34], allows us to find quasiblack holes with pressure using the hydrostatic equilibrium equation. Interestingly the Schwarzschild interior solution has a constant energy density and, numerically, the Buchdahl limit is attained by taking the limit of infinite central pressure. Namely, the Schwarzschild stars satisfy $R / M>9 / 4$, with the upper bound for the most compact Schwarzschild star, the Buchdahl limit $R / M=9 / 4$, being found by taking the limit $p_{c} \rightarrow \infty$ in the solution. We then expect to find quasiblack holes in a similar situation, i.e, for static charged fluid spheres with polytropic equation of state in the limit of very large polytropic exponent. This was investigated numerically and the more important results are presented in the next sections.

\subsubsection{The Buchdahl limit}

In order to see more clearly the extremely compact limit of the objects studied in the present work we plot in Fig. 8 an amplified version of Fig. 7, showing the limit of $R / M$ for large $\gamma$. The aim of such a figure is to show the ratio $R / M$ for the uncharged case $(\alpha=0.0)$ and for the largest considered charge fraction $(\alpha=0.99)$ in the high polytropic exponent regime.

In the uncharged case, $\alpha=0.0$, the smallest value of $R / M$ we have found is approximately 2.27 , which is very close to the Buchdahl bound, $R / M=9 / 4=2.25$ [13]. The Schwarzschild interior solution is probably the simplest case where the Buchdahl bound can be verified. In fact, if the central pressure is allowed to be infinite then the bound $R / M=9 / 4$ is reached. In such a limit, the Schwarzschild interior solution corresponds to an incompressible fluid (constant energy density) with a monotonically decreasing pressure whose central value is arbitrarily large.

In the charged $\alpha=0.99$ case, the numerical result is also very close to the extremal bound of Andréasson for the compactness of charged static spheres [29]. In fact, from Table II one gets $R / M=1.03$ and $R / R_{+}=$ 1.01 , indicating that the radius of the charged sphere is really very close to its own gravitational radius. This confirms the fact that the analogous to the Buchdahl limit for charged static spherical objects is the quasiblack hole limit, $R / M=R_{+} / M=Q / M=1$ (see below).

\subsubsection{The quasiblack hole limit}

As shown in Figs. 7 and 9, there are values of $\alpha$ and $\gamma$ for which we find $R / M \approx 1.02676$ and $Q / M \approx 0.999793$, in other words $R \approx M \approx Q$, indicating that the quasiblack hole limit is about to be reached. The best values we have found, i.e., the ones whose corresponding solution is the closest to the quasiblack hole solution, are obtained by considering the charge fraction $\alpha=0.99$ and the polytropic exponent $\gamma=17.0667$. For higher values of $\alpha$ or $\gamma$, our numerical code fails to converge. Therefore, the values $\alpha=0.99$ and $\gamma=17.0667$ were chosen as best values and the other functions and properties of the corresponding solution were determined.

Following [36], given a static spherically symmetric spacetime solution one has to check also the behavior of the metric functions in order to decide if the solution is really a quasiblack hole, or, at least, really close to one. For that we have studied the behavior of $A(r)$ and $B(r)$ for large $\gamma$ and large $\alpha$.

We plot the metric function $A^{-1}(r)$ against the radial coordinate in Fig. 12, for the charge fraction $\alpha=0.99$, polytropic exponents $\gamma=4 / 3$ and $\gamma=17.0667$, and central density $\rho_{c}=10 \rho_{0}=1.78266 \times 10^{16} \mathrm{~kg} / \mathrm{m}^{3}$. Note that $A^{-1}(r)$ decreases monotonically with $r$, so that its 


\begin{tabular}{cccccccc}
\hline \hline & $\alpha$ & $M \times 10^{5}[\mathrm{~m}]$ & $Q \times 10^{5}[\mathrm{~m}]$ & $R \times 10^{5}[\mathrm{~m}]$ & $R / M$ & $R / R_{+}$ & $R / R_{-}$ \\
\hline 1 & 0.50 & 1.22295 & 0.879711 & 2.32188 & 1.89859 & 1.12033 & 6.21802 \\
2 & 0.70 & 1.47248 & 1.31759 & 2.33518 & 1.58588 & 1.09640 & 2.86491 \\
3 & 0.90 & 1.92775 & 1.90301 & 2.34444 & 1.21615 & 1.04868 & 1.44728 \\
4 & 0.99 & 2.27478 & 2.27431 & 2.33566 & 1.02676 & 1.00631 & 1.04807 \\
\hline \hline
\end{tabular}

TABLE II: The obtained values of the mass, charge and radius of the charged star, in geometric units, with the corresponding values of $R / M, R / R_{+}$and $R / R_{-}$, for several charge fractions $\alpha$ in the case $\rho_{c}=1.78266 \times 10^{16} \mathrm{~kg} / \mathrm{m}^{3}$ and $\gamma=17.0667$.

minimum value is found at the surface of the sphere. In the case considered here, the minimum value of $A^{-1}(r)$ is $4.05648 \times 10^{-4}$, indicating that we are close to the quasiblack hole limit for such a metric function, i.e., $A^{-1}(r=R)=\epsilon$ at the surface of the object, $r=R$.
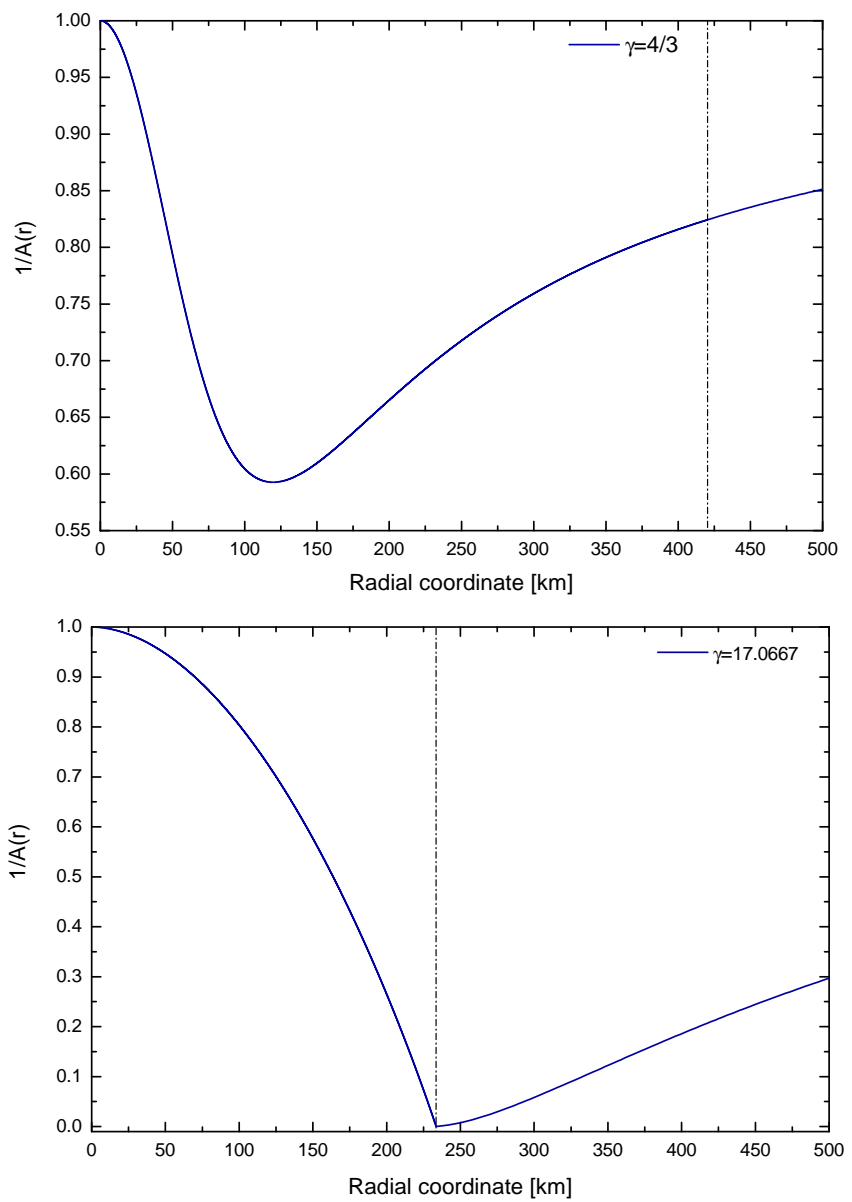

FIG. 12: The metric function $A^{-1}(r)$ as a function of the radial coordinate for $\gamma=4 / 3$ (top) and $\gamma=17.0667$ (bottom). In both cases $\alpha=0.99$. The vertical dashed line indicates the surface of the star.

In Fig. 13 we plot the metric function $B(r)$ as a function of the radial coordinate, for the charge fraction $\alpha=0.99$, polytropic exponents $\gamma=4 / 3$ and $\gamma=17.0667$, and central density $\rho_{c}=10 \rho_{0}=1.78266 \times 10^{16} \mathrm{~kg} / \mathrm{m}^{3}$. Once the values of $M, Q$ and $R$ are already known, this graphic is drawn by solving the conservation equation (13), integrating from the surface until the center
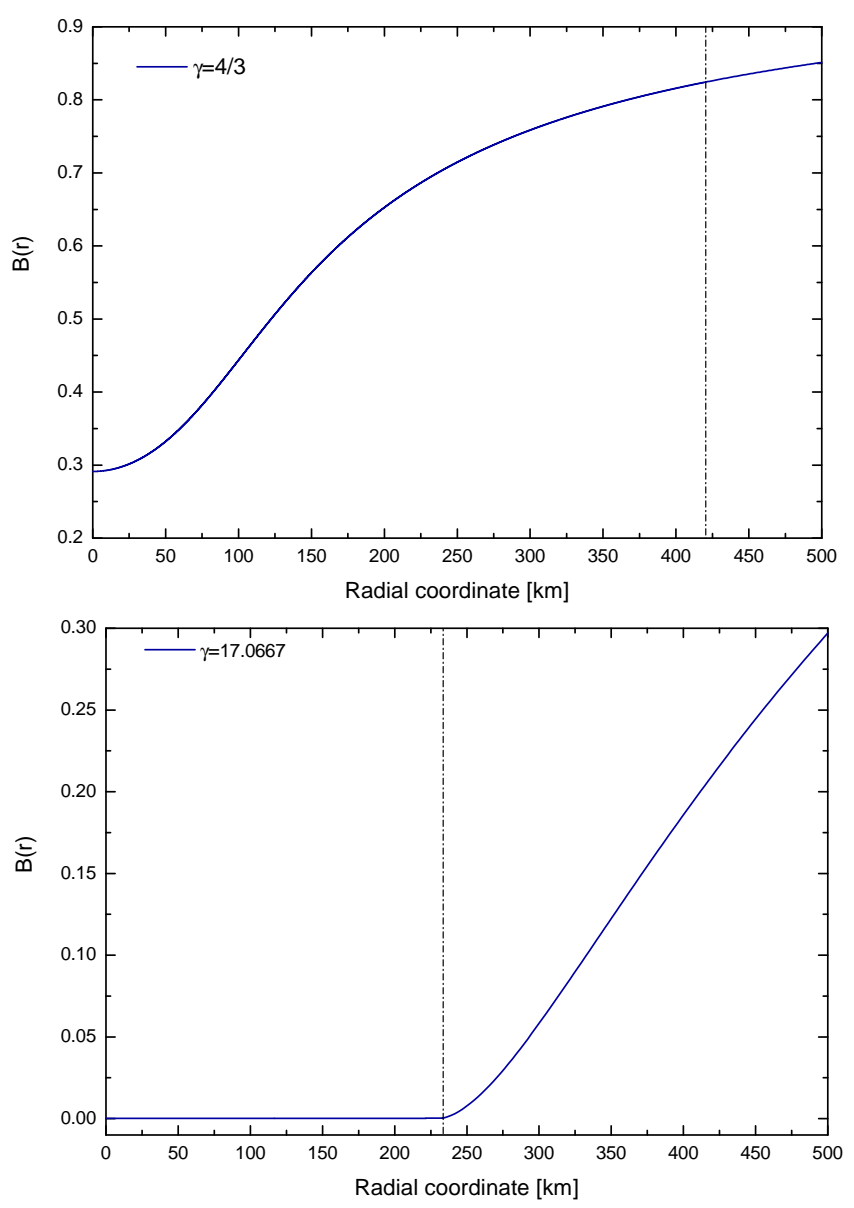

FIG. 13: The metric function $B(r)$ as a function of the radial coordinate for $\gamma=4 / 3$ (top) and $\gamma=17.0667$ (bottom). In both graphs we used $\alpha=0.99$. The dashed vertical line represents the surface of the sphere.

of the sphere. For these particular values of parameters, the metric function $B(r)$ assumes very small values, increasing slowly with the radial coordinate, to reach the largest value at the surface of the star, where it equals the metric coefficient $A^{-1}(r)$. The maximum value is $B(r)=4.05648 \times 10^{-4}$ at $r=R$. As just said, this value is the same as the value of $A^{-1}(R)$, because at the surface of the star one has $B(R)=A^{-1}(R)=1-\frac{2 M}{R}+\frac{Q^{2}}{R^{2}}$, which is due to the fact that interior solution is matched smoothly to the Reissner-Nordström exterior solution. It is worth noticing that the metric function $B(r)$ is very 
small all along the interior of the sphere, also indicating that the solution is close to the quasiblack hole limit in which $B(r)$ is vanishingly small, $B(r) \rightarrow \epsilon$ across the hole region inside matter.

The ratio $Q / M$ as a function of the charge fraction $\alpha$, for the polytropic exponent $\gamma=17.0667$, and for the central density $\rho_{c}=1.78266 \times 10^{16} \mathrm{~kg} / \mathrm{m}^{3}$ has been shown in Fig. 9. As can be seen in that figure, the ratio $Q / M$ approaches one with the increasing charge fraction. For $\alpha=0.99$, the values of the charge and of the mass of the given star are very close to each other $(Q \approx M)$, which implies that $R_{ \pm} \equiv M \pm \sqrt{M^{2}-Q^{2}} \approx M$, where $R_{ \pm}$are, respectively, would be event horizon and the Cauchy horizon of the corresponding star, found as usual through the solutions of the equation $B(R)=0$. Moreover, from the values of ratios $Q / M$ and $R / M$, for the charge fraction $\alpha=0.99$ and $\gamma=17.0667$, we conclude that the radius of the charged sphere is larger than the radius of the event horizon of the corresponding Reissner-Nordström black hole, $R_{+}$, indicating that we have a static equilibrium configuration. Also, since $R / R_{+} \approx 1$, the boundary of the star approaches its own gravitational radius, which, together with the other properties found above, indicates that the quasiblack holes limit is about to be reached, see also [22, 23] and [34].

The values of $M, Q, R, R_{ \pm}$and their relations are shown in Table II for the maximum $\alpha$ and $\gamma$ that permit good numerical results, namely, $\alpha=0.99$ and $\gamma=$ 17.0667. We obtain $Q \approx M$, in geometric units, and then $R \simeq R_{+} \simeq R_{-}$. These results, together with the fact that $B(r) \sim \epsilon$ for all $0 \leq r \leq R$ and $A^{-1}(R) \sim \epsilon($ small $\epsilon)$ guarantee that we are close to the quasiblack hole limit (see [36] for a precise definition a quasiblack hole).

As well known, similar extreme relations characterizing quasiblack holes, such as $R_{+} \simeq R_{-} \simeq M=Q$, are found in the case of charged dust stars, with zero interior pressure $(p=0)$. This follows mainly in charged systems that satisfy the Majumdar-Papapetrou conditions, see, e.g, [35]. The present result is an additional example of quasiblack holes with pressure, as the one found in Ref. [34] (see also [37]).

\subsection{Speed of sound within the fluid and the causality condition}

If one is interested in restricting the charged sphere solutions to those that do not violate causality, the speed of sound inside the fluid is an important property to investigate.

The speed of sound is defined through the equation $c_{s}^{2}=d p / d \rho$. So from the equation of state (16) one finds

$$
c_{s}^{2}=\frac{d p}{d \rho}=\gamma \frac{p}{\rho}=\omega \gamma \rho^{\gamma-1} .
$$

As seen from Eq. (21), the speed of sound gets larger than the speed of light if

$$
\omega \gamma \rho^{\gamma-1}>1
$$

For the charged polytropic fluid we are considering here, the energy density $\rho(r)$ decreases towards the surface of the star and so does the speed of sound. This is true for finite $\gamma$. In other words, for a given equilibrium solution, the speed of sound is greatest at the center of the star. This has been confirmed numerically for all values of the parameters we have checked. Hence to test the causality condition, $c_{s} \leq 1$, it is sufficient to determine the speed of sound at the center of the star.

It is clear from Eq. (21) that, for relatively small energy densities and small polytropic exponents, $c_{s}^{2}$ is smaller than unity. However, as the polytropic index grows, the fluid gets stiffer and eventually becomes incompressible for $\gamma \rightarrow \infty$. Hence, for given values of the polytropic constant $\omega$ and central energy density $\rho_{c}$, there is a particular value of $\gamma$ above which causality is violated. In the case of Fig. 14 it is $\gamma \simeq 3.3$.

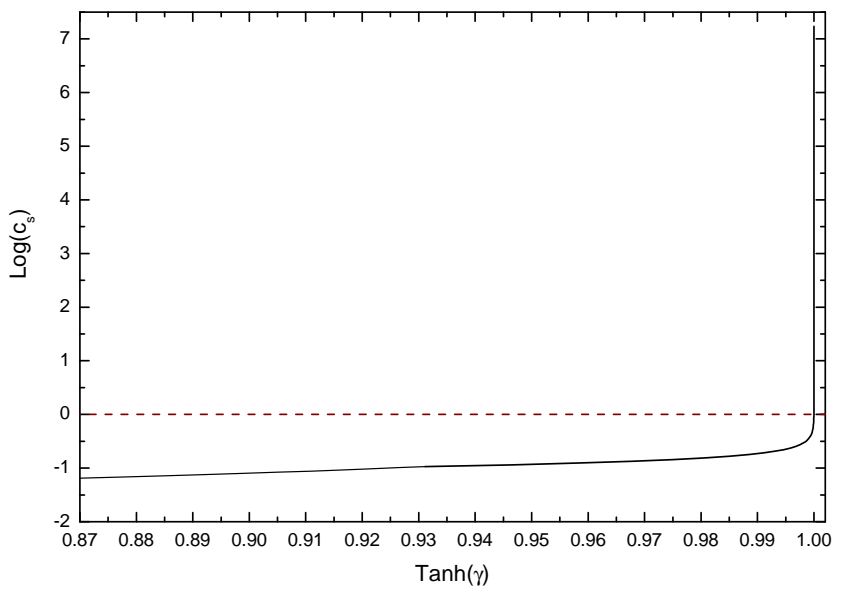

FIG. 14: Speed of sound in the center of the star against the polytropic exponent $\gamma$ for the central density $\rho_{c}=1.78266 \times$ $10^{16} \mathrm{~kg} / \mathrm{m}^{3}$. For $\gamma \gtrsim 3.3$ the speed of sound $c_{s}$ is larger than the speed of light.

In the graphic of Fig. 14 we plot the speed of sound at the center of the star $\left(c_{s}\right)$ as a function of the polytropic exponent $(\gamma)$ for the central energy density $\rho_{c}=$ $1.78266 \times 10^{16} \mathrm{~kg} / \mathrm{m}^{3}$. It is seen that for large values of $\gamma$ the speed of sound exceeds the speed of light. This is because as $\gamma$ grows very large, the value of $\rho(r)$ approaches a constant and $d p / d \rho$ becomes arbitrarily large. The speed of sound strongly depends on the central energy density, and increases with the exponent $\gamma$. In the limit of very large $\gamma$ the charged spheres become incompressible for which the sound speed cannot be defined by Eq. (21). In such a limit, compact uncharged spheres can be found, these being the interior Schwarzschild solution with the most compact one given by the Buchdahl limit. In the charged case the limiting $\gamma \rightarrow \infty$ solution is again a $\rho(r)=$ constant solution and it yields the quasiblack hole limit. 


\section{CONCLUSIONS}

We have studied electrically charged polytropic spheres in the context of Einstein-Maxwell theory. The spheres contain a spherically symmetric distribution of charged perfect fluid, and the exterior spacetime is represented by the Reissner-Nordström metric. The charge density $\rho_{e}$ and the energy density $\rho$ were assumed to have the relation $\rho_{e}=\alpha \rho$, whereas the fluid assumes a polytropic equation of state relating the pressure $p$ and the energy density $\rho$ of the fluid, $p=\omega \rho^{\gamma}$, with $\omega$ and $\gamma$ being constants. The choice of parameter $\omega$ is such that, for $\gamma=5 / 3$ and appropriate central energy density $\rho_{c}$, the system is close to the realistic neutron stars. We have studied the Oppenheimer-Volkoff limit, the Buchdahl limit and the quasiblack hole limit.

We have analyzed the configurations for several $\gamma$, from $\gamma=4 / 3$ to $\gamma$ reasonably high. Indeed, we have found that for a central energy density that is ten times larger than the normalization factor the highest value of the polytropic exponent that produced proper numerical results is $\gamma=17.0667$. In such a case, the numerical results approached interesting limiting cases.

In the zero charge case, $\alpha=0.0$, for all of the central energy densities considered, in the limit of large polytropic exponents we found solutions for stars that are very close to the Buchdahl limit, $R / M=9 / 4$. The mass to radius relation increases with the polytropic exponent attaining a value very close to $9 / 4$ for $\gamma=17.0667$. In such a limit the spheres are similar to the Schwarzschild star since they have a constant energy density, the central pressure in this limit being arbitrarily large.

For the charged case, for fixed finite $\gamma$, and varying the central pressure we have not found quasiblack holes. On the other hand, in the limit of very high $\gamma$, and with the central pressure tending to infinity, we have shown that the quasiblack hole limit is reached. In fact, with increasing polytropic exponent and charge fraction the relation $R / M$ approaches unity. The largest value of such a ratio is found considering $\alpha=0.99$ and $\gamma=17.0667$, which are the highest values our numerical code furnished trustworthy results. For these values of $\alpha$ and $\gamma$ we found also that $Q \simeq M$, and so the radius of the sphere is close to the corresponding horizon radius $R \simeq R_{+}=$ $M+\sqrt{M^{2}-Q^{2}}$. In addition to this we verified other properties of the charged spheres that indicate beyond doubt the presence of quasiblack holes with pressure.

The physical properties of charged spheres with other choices of equations of state and other charge density profiles should also be investigated, we shall report such analyses in a future work.

\section{Acknowledgments}

JDVA thanks Fundação Universidade Federal do ABC - UFABC, Brazil, and Coordenação de Aperfeiçoamento de Pessoal de Nível Superior - CAPES, Brazil, for a grant. VTZ would like to thank Conselho Na- cional de Desenvolvimento Científico e Tecnológico CNPq, Brazil, for grants, and Fundação de Amparo à Pesquisa do Estado de São Paulo for a grant (Processo 2012/08041-5). JPSL thanks the support of the Fundação para a Ciência e a Tecnologia of Portugal - FCT, projects PTDC/FIS/098962/2008 and PEstOE/FIS/UI0099/2011. JPSL and VTZ thank the Observatório Nacional do Rio de Janeiro - ON, Brazil, for hospitality.

\section{Appendix A: Dimensionless relativistic equations of a polytrope}

For the numerical calculations, the relativistic equations of a polytrope must be written in dimensionless form. For this, we introduce the dimensionless radial coordinate $\varepsilon$ given by

$$
\varepsilon=r \sqrt{4 \pi \rho_{c}},
$$

and the new variables $v(\varepsilon), u(\varepsilon)$, and $\theta(\varepsilon)$ defined by

$$
\begin{gathered}
v(\varepsilon)=\sqrt{4 \pi \rho_{c}} m(r), \\
u(\varepsilon)=\frac{\sqrt{4 \pi \rho_{c}}}{\varepsilon^{2}} q(r), \\
\theta(\varepsilon)=\left(\frac{\rho(r)}{\rho_{c}}\right)^{\gamma},
\end{gathered}
$$

$\rho_{c}$ being the central energy density of the star. In terms of the normalized energy density $\theta$, the pressure becomes $p(r)=\omega \rho_{c}^{\gamma} \theta(\varepsilon)$. Considering the dimensionless variables $u, v, \theta, B$ and the relation (17), the relativistic equations of a polytrope, Eqs. (8), (12), and (14) in a dimensionless form are

$$
\begin{aligned}
\frac{d u}{d \varepsilon}=-\frac{2 u}{\varepsilon}+\frac{\alpha \theta^{1 / \gamma}}{\sqrt{1-\frac{2 v}{\varepsilon}+\varepsilon^{2} u^{2}}}, \\
\frac{d v}{d \varepsilon}=\theta^{1 / \gamma} \varepsilon^{2}+\frac{\alpha \varepsilon^{3} \theta^{1 / \gamma} u}{\sqrt{1-\frac{2 v}{\varepsilon}+\varepsilon^{2} u^{2}}}, \\
\frac{d \theta}{d \varepsilon}=-\varepsilon\left(\theta+\frac{\left.\rho_{c}^{1-\gamma} \theta^{1 / \gamma}\right)}{\omega}\left(\frac{\omega \rho_{c}^{\gamma-1} \theta-u^{2}+\frac{v}{\varepsilon^{3}}}{1-\frac{2 v}{\varepsilon}+\varepsilon^{2} u^{2}}\right)\right. \\
+\frac{\alpha \rho_{c}^{1-\gamma} \omega^{-1} u \theta^{1 / \gamma}}{\sqrt{1-\frac{2 v}{\varepsilon}+\varepsilon^{2} u^{2}}}
\end{aligned}
$$

This gives a set of three coupled differential equations, (A5)-(A7), that are solved simultaneously to get the equilibrium solutions. The boundary conditions adopted in the center of the star, where $\varepsilon=0$, are $v(0)=0$, $u(0)=0$ and $\theta(0)=1$. The maximum value of $\varepsilon$ is found when $\theta(\varepsilon)=0$, and such a particular value of $\varepsilon$ is identified as the radius of the polytropic sphere, $\varepsilon=\varepsilon_{s}$,

In astrophysics of neutron stars, units appropriate to nuclear physics [15] are usually used, so that, for units 
in which the speed of light is set to unity $(c=1)$, the pressure $p$ and energy density $\rho$ are given in $\mathrm{MeV} / \mathrm{fm}^{3}$. Moreover, from the relation (17), the charge density $\rho_{e}$ is also measured in $\mathrm{MeV} / \mathrm{fm}^{3}$. The reference energy density, $\rho_{0}$, used as a normalization factor in the numerical calculations, is $\rho_{0}=1.0 \mathrm{MeV} / \mathrm{fm}^{3}$. Transforming to MKS units, which we use in this paper, this means $\rho_{0}=1.78266 \times 10^{15} \mathrm{~kg} / \mathrm{m}^{3}$. The normalization factor may be changed according to the region of the central energy density one is interested, or depending on the equation of state of the fluid being used. We have done both variations, but in this paper we report the results obtained by using the TOV equation normalized in terms of the central energy density $\rho_{c}$, as given by Eqs. (A1), (A2), (A3) and (A4).

In the present analysis, the polytropic constant $\omega$ is normalized in terms of the reference central energy density $\rho_{0}=1.0 \mathrm{MeV} / \mathrm{fm}^{3}$. The particular value $\omega=$ $1.47518 \times 10^{-3}\left[\mathrm{fm}^{3} / \mathrm{MeV}\right]^{\gamma-1}$, that is equivalent to $\omega=$ $1.47518 \times 10^{-3}\left(1.78266 \times 10^{15}\right)^{1-\gamma}\left[\frac{\mathrm{m}^{3}}{\mathrm{~kg}}\right]^{\gamma-1}$, was chosen for the sake of comparison to the results of previous works [24]. Notice that, with this choice, the parameter $\omega$ results to be a function of the polytropic exponent, $\omega=\omega(\gamma)$.

The integration of equations (A5), (A6) and (A7) is stopped at the point $\varepsilon$ where the pressure $\theta(\varepsilon)$ reaches negative values, or otherwise, when it gets smaller than an appropriate chosen value, $\theta(\varepsilon) \sim 0$. The corresponding vale of the radial coordinate $\varepsilon=\varepsilon_{s}$ is extracted and the radius of the sphere is obtained from the relation $R=\frac{\varepsilon_{s}}{\sqrt{4 \pi \rho_{c}}}$. Then, the physical quantities, mass $M$ and charge $Q$, are calculated respectively from $M \equiv m(R)=\frac{v\left(\varepsilon_{s}\right)}{\sqrt{4 \pi \rho_{c}}}$ and $Q \equiv q(R)=\frac{\varepsilon_{s}^{2} u\left(\varepsilon_{s}\right)}{\sqrt{4 \pi \rho_{c}}}$.

After obtaining $v(\varepsilon)$ and $u(\varepsilon)$ the metric functions $B(\varepsilon)$ and $A(\varepsilon)$ are determined from the relations

$$
\begin{aligned}
& \frac{d B(\varepsilon)}{d \varepsilon}=2 \varepsilon B(\varepsilon)\left(\frac{\omega \rho_{c}^{\gamma-1} \theta(\varepsilon)-u^{2}(\varepsilon)+\frac{v(\varepsilon)}{\varepsilon^{3}}}{1-\frac{2 v(\varepsilon)}{\varepsilon}+\varepsilon^{2} u^{2}(\varepsilon)}\right) \\
& A^{-1}(\varepsilon)=1-\frac{2 v(\varepsilon)}{\varepsilon}+\varepsilon^{2} u^{2}(\varepsilon) .
\end{aligned}
$$

[1] A. S. Eddington, The internal constitution of stars, (Cambridge University Press, Cambridge, 1926).

[2] S. Chandrasekhar, "The highly collapsed configurations of a stellar mass", Mon. Not. R. Astron. Soc. 91, 456 (1931).

[3] S. Chandrasekhar, "The highly collapsed configurations of a stellar mass (second paper)", Mon. Not. R. Astron. Soc. 95, 207 (1935).

[4] R. C. Tolman, "Static solution of Einstein's field equation for spheres of fluid", Phys. Rev. 55, 364 (1939).

[5] J. R. Oppenheimer and G. Volkoff, "On massive neutron cores", Phys. Rev. 55, 374 (1939).

[6] L. D. Landau, "On the theory of stars", Physikalische Zeitschrift der Sowjetunion 1, 285 (1932).

[7] J. R. Oppenheimer and H. Snyder, "On Continued Gravitational Contraction", Phys. Rev. 56, 455 (1939).

[8] C. W. Misner and D. H. Sharp, "Relativistic Equations for Adiabatic, Spherically Symmetric Gravitational Collapse", Phys. Rev. 136, B571 (1964).

[9] C. W. Misner, "Gravitational collapse", in Astrophysics and General Relativity (11th Brandeis Summer Institute in Theoretical Physics), eds. M. Chrétien, S. Deser, and J. Goldstein (Gordon and Breach, New York, 1969).

[10] B. K. Harrison, K. S. Thorne, M. Wakano, and J. A. Wheeler, Gravitation Theory and Gravitational Collapse, (University of Chicago Press, Chicago, 1965).

[11] K. S. Thorne, "Relativistic Stellar Structure and Dynamics", in High Energy Astrophysics, Volume III (1966 Les Houches Summer School of Theoretical Physics), eds. C. DeWitt, E. Schatzmann, P. Véron (Gordon and Breach, New York, 1967).

[12] R. F. Tooper, "General relativistic polytropic fluid spheres", Astrophys. J. 140, 434 (1964).

[13] H. A. Buchdahl, "General Relativistic Fluid Spheres", Phys. Rev. 116, 1027 (1959).
[14] S. Chandrasekhar, An Introduction to the Study of Stellar Structure (University of Chicago Press, Chicago, 1939).

[15] N. K. Glendenning, Compact Stars: Nuclear Physics, Particle Physics, and General Relativity (SpringerVerlag, Heidelberg, 1996).

[16] Y. B. Zel'dovich and I. D. Novikov, Relativistic Astrophysics 1: Stars and Relativity, (University of Chicago Press, Chicago 1971), (translation from the 1967 Russian edition).

[17] C. W. Misner, K. S. Thorne, and J. A. Wheeler, Gravitation, (Freeman, San Francisco, 1973).

[18] K. A. Bronnikov and S. G. Rubin, Black Holes, Cosmology and Extra Dimensions (World Scientific, Singapore, 2013).

[19] W. Israel, "Dark stars: the evolution of an idea", in 300 years of gravitation, eds. S. W. Hawking and W. Israel (Cambridge University Press, 1987), p. 199.

[20] J. Bekenstein, "Hydrostatic equilibrium and gravitational collapse of relativistic charged fluid balls", Phys. Rev. D 4, 2185 (1971).

[21] J. L. Zhang, W. Y. Chau, and T. Y. Deng, "The influence of a net charge on the critical mass of a neutron star", Astrophys. Space. Sci. 88, 81 (1982).

[22] F. de Felice, Y. Yu, and J. Fang, "Relativistic charged spheres", Mon. Not. R. Astron. Soc. 277, L17 (1995).

[23] F. de Felice, L. Siming, and Y. Yunqiang, "Relativistic charged spheres: II. Regularity and stability", Classical Quantum Gravity 16, 2669 (1999); arXiv:gr-qc/9905099.

[24] S. Ray, A. L. Espíndola, M. Malheiro, J. P. S. Lemos, and V. T. Zanchin, "Electrically charged compact stars and formation of charged black holes", Phys. Rev. D 68, 084004 (2003); arXiv:astro-ph/0307262.

[25] C. Ghezzi, "Relativistic structure, stability, and gravitational collapse of charged neutron stars", Phys. Rev. D 
72, 104017 (2005); arXiv:gr-qc/0510106.

[26] B. B. Siffert, J. R. de Mello, and M. O. Calvão, "Compact charged stars", Brazilian J. Phys. 37, 2B (2007).

[27] Y. Q. Yu and S. M. Liu, "Relativistic charged balls", Commun. Theor. Phys. 33, 571 (2000); arXiv:grqc/9904050.

[28] M. K. Mak, P. N. Dobson, and T. Harko, "Maximum mass-radius ratios for charged compact general relativistic objects", Europhys. Lett. 55, 310 (2001); arXiv:grqc/0107011.

[29] H. Andréasson, "Sharp bounds on the critical stability radius for relativistic charged spheres", Commun. Math. Phys. 288, 715 (2009); arXiv:0804.1882 [gr-qc].

[30] W. B. Bonnor, "Gravitational collpase", Nature 204, 868 (1964).

[31] W. B. Bonnor and S. B. P. Wickramasuriya, "Are very large gravitational redshifts possible?", Mon. Not. R. Astron. Soc. 170, 643 (1975).

[32] J. P. S. Lemos and V. T. Zanchin, "Bonnor stars in d spacetime dimensions", Phys. Rev. D 77, 064003 (2008); arXiv:0802.0530 [gr-qc].

[33] J. P. S. Lemos and E. Weinberg, "Quasiblack holes from extremal charged dust", Phys. Rev. D 69, 104004 (2004); arXiv:gr-qc/0311051.

[34] J. P. S. Lemos and V. T. Zanchin, "Quasiblack holes with pressure: relativistic charged spheres as the frozen stars",
Phys. Rev. D 81, 124016 (2010); arXiv:1004.3574 [gr-q].

[35] J. P. S. Lemos, "Black holes and quasiblack holes: Some history and remarks" Uch. Zap. Kazan. Univers. 153, 215 (2011); arXiv:1112.5763 [gr-qc].

[36] J. P. S. Lemos and O. B. Zaslavskii, "Quasiblack holes: definition and general properties", Phys. Rev. D 76, 084030 (2007); arXiv:0707.1094 [gr-qc].

[37] J. P. S. Lemos and O. B. Zaslavskii, "Quasiblack holes with pressure: General results", Phys. Rev. D 82, 024029 (2010); arXiv:11011.2768 [gr-qc].

[38] R. Meinel and M. Hütten, "On the black hole limit of electrically counterpoised dust configurations", Classical Quantum Gravity 28, 225010 (2011); arXiv:1105.3807 [gr-qc].

[39] J. P. S. Lemos and V. T. Zanchin, "Regular black holes: Electrically charged solutions, Reissner-Nordström outside a de Sitter core", Phys. Rev. D 83, 124005 (2011); arXiv:1104.4790 [gr-qc].

[40] J. P. S. Lemos and P. Pani, "Gravitational fields with sources, regular black holes, quasiblack holes, and analogue black holes (Session AT3 of the 13th Marcel Grossmann Meeting)", Proceedings of the 13th Marcel Grossmann Meeting, eds. Jantzen et al, to appear; arXiv:13xx.xxxx [gr-qc], to appear. 